\title{
A Novel Technique of Piezoelectric Energy Harvesting
}

\author{
Burri Ankaiah, Rajashekar P. Mandi, Sujo Oommen, Aditya Balllaji, K. Narayana Swamy
}

\begin{abstract}
Energy harvesting is the technology to extract energy from environment with many surrounding sources of energy. From these sources it is used to extract less electrical power energy and boost up tiny electrical systems or amount of energy stored in a battery. Many methods in energy harvesting among one of the method for harvesting energy is piezoelectric transducers. Energy harvesting depends upon so many factors like conducting circuit, number of sensors, and coupling coefficient of piezoelectric sensors with electromechanical. For large scale applications, one of the best suited technique energy harvesting .
\end{abstract}

Keywords : Converter circuit, Energy harvesting, Sound buzzers, sensor piezoelectric transducer, storage device/load, Scheme of arrangement.

\section{INTRODUCTION}

At present scenario the universe is shifting for new technique sources of energy other than fossil fuels and sources of the non-renewable systems. The energy can neither be created, nor be destroyed [1] change of one form to another form of the energy. The lost energy in the form of mechanical force, vibrations or stress can be harvested with the transducer in the piezoelectric. Human beings spend energy to routine activities like as jumping, walking, running etc. This kind of lost energy can be harvested with vibrations created in shoes or on the floor [5-6]. This kind of energy which can be used for charging mobile phone or storage energy in the battery, energy harvesting can be implemented with circuitry. By applying stress to transducer energy harvested . Piezoelectric transducer act as sound buzzer for mechanical vibrations, a sensor is used to extract energy[2].There are many techniques available at present scenario for conversation of vibration with mechanical energy to electrical energy of which the most of methods are electromagnetic, piezoelectric conversion and electrostatic. Rather than exhausting batteries [3] the portable devices have their own Power sources. The lost energy can be obtained with piezoelectric material due to vibration of pedestals, rail, runway, roadway, mobile floor etc. Continues strain or vibration energy which is wasted that can be transformed into useful electrical energy for low power electrical and

Revised Manuscript Received on March 5, 2020.

* Correspondence Author

Burri Ankaiah*, Assistant Professor,School of EEE, REVA University/,Bangalore, India. Email: burriankaiah@reva.edu.in

Dr. Rajashekar P.Mandi, Professor\&Director,School of EEE, REVA University, Banglore, India. Email: dir.eee@ reva.edu.in

K. NarayanSwamy,Associate Professor,School of EEE,REVA University, Banglore, India. Email: knarayanswamy@reva.edu.in

Sujo Oommen, Associate Professor,School of EEE,REVA University, Banglore, India.

Aditya Balllaji, Associate Professor,School of EEE,REVA University, Banglore, India. electronics devices [5]. From vibrations created electrical Energy with the help of walking, jumping, running etc. The square type piezoelectric diaphragm is used to harvest this kind of energy and also it can be rectified through a bridge full wave rectifier. The rectifier output is amplification can be done through DC-DC boost converter which is connected to load.

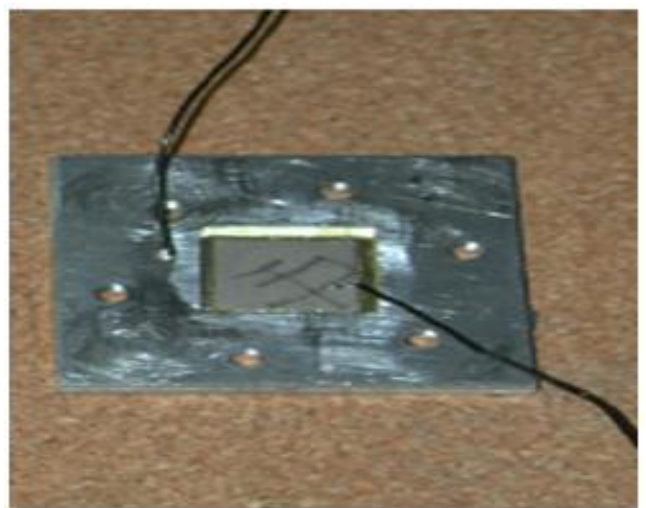

Fig.1 Square type piezoelectric diaphragm

\section{PIEZOELECTRIC TRANSDUCER}

One of the classes of material from ferroelectric is piezoelectric, which has exhibiting dipole electric and charge separation. Due to heating effect Curie temperature is created and a very strong electric filed can be applied. Even the material is cooled also it will remains its orientation. The production of sound in mechanical vibrations can seen in converse piezoelectric effect with electric potential applied to it. This kind of piezoelectric application can be used as sound buzzers. When input is mechanical vibrations is due to piezoelectric effect it produces electrical potential [2]. This kind of effects which makes application in the sensor and also for harvesting energy for both effects (direct and converse) piezoelectric transducer which has two equations.

Piezoelectric effect direct : $\{D\}=[e] T\{S\}+\left[\alpha^{S}\right]\{E\}$

Piezoelectric effect Converse: $\{T\}=\left\{C^{E}\right\}\{S\}-[e]\{E\}$ Where

$\left[C^{E}\right]$ : At constant electric field strength in Matrix of elastic coefficients

$[e]:$ permittivity dielectric matrix

$\{E\}$ : vector of electric field

$\left[\propto^{S}\right]$ :constant material strain in dielectric matrix

$\{S\}$ : strain vector

$\{D\}$ : vector of electric displacement [3] 


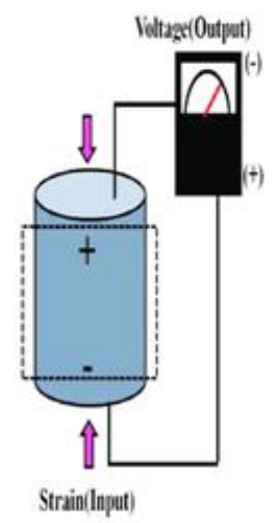

Diret Piezvelectric Effict

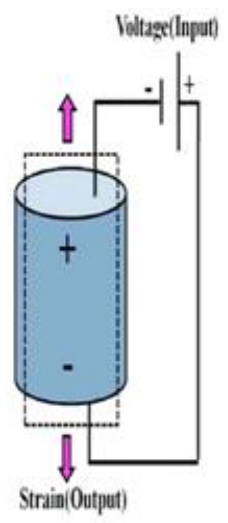

Coeverse Pikzodectric Effect

Fig.2 Decompression and Compression of PZT forming AC signal

The AC signal is generated in transducer as an input when fed with vibrations of mechanical [1]. PZT generates an AC signal with changing amplitude electrical signal. The positive half of the AC signal is created in deformed. In negative half of the AC signal is created in formed back to orientation it is demonstrated in Fig. 2.The maximum charge is induced in transducer under self resonant frequency stressed range [3].

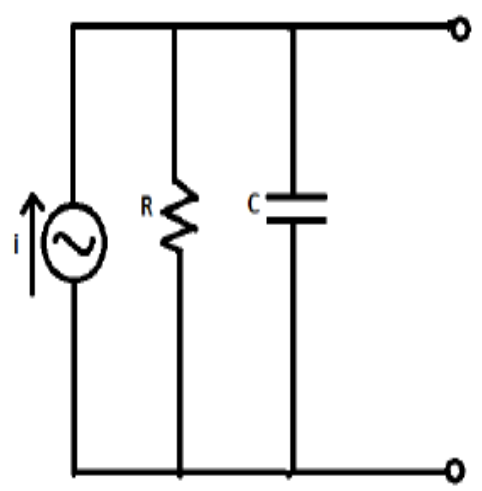

Fig.3 Equivalent circuit for piezoelectric

As shown in Fig.3 the equivalent circuit for piezoelectric transducer with high resistance connected in parallel and also capacitance in parallel and imagined with AC current source.

The Helmholtz's equation frequency for self resonant in piezoelectric is calculated

$$
f_{0=C / 2 \pi \frac{\sqrt{4 a^{2}}}{d(t+k a)}}
$$

Where is K Material constant, $d$ is Diameter of the support, a is Radius of ceramic diaphragm $(\mathrm{cm}), \mathrm{C}$ is Velocity of energy wave and $\mathrm{fO}$ is Resonant frequency $(\mathrm{Hz})$

\section{ANALYSIS AND DESIGN}

\section{A. Design for Harvester Model}

he energy generated study is used with piezoelectric harvester. The design of harvester model in block diagram in Fig. 4.

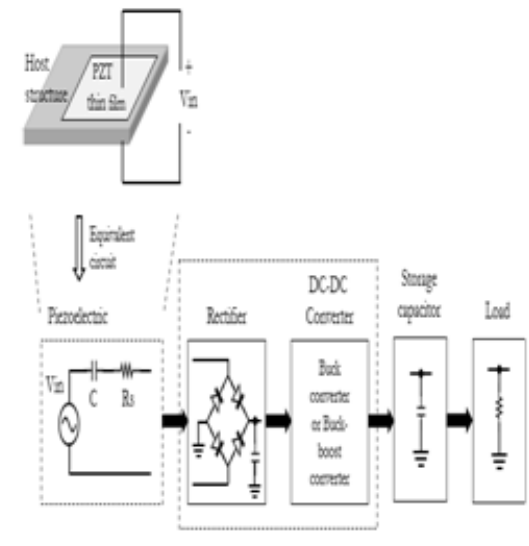

Fig.4 Working flow of Harvester Model Blocks
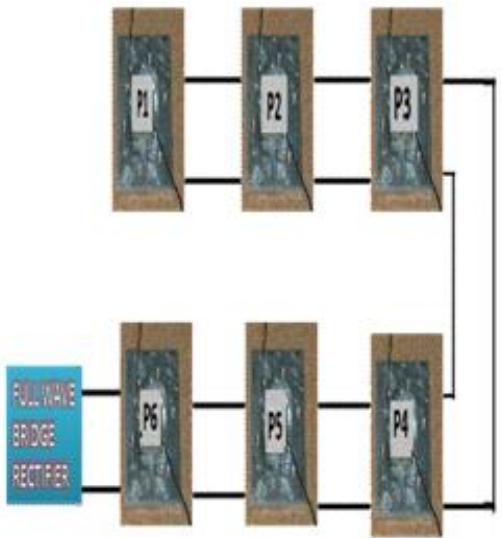

Fig.5 Elements of parallel connections

Next step in energy generation is full wave bridge rectifier which converts AC signal from the block of piezoelectric cell to DC signal so it can be connected to load or stored. For full wave bridge rectifier 4 pin IC W10M is used [7] with thin voltage drop is created these can be reduced by using dedicated IC LTC $® 3588-1[9]$ or low drop diodes. For comparative very high levels the DC-DC boost circuit is used. The system integral part as the harvested energy is low power signal.

\section{MATH}

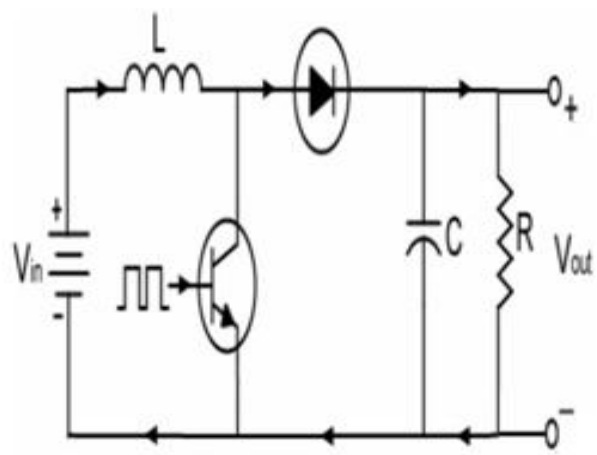

Fig.6 Circuit for DC-DC Boost converter

Circuit for DC-DC boost converter is shown in Fig. 6.The generated output of DC-DC boost converter circuit is directly connected to load. 


\section{EXPECTED DESIGN FLOW IN PARALLEL CONNECTION}

In Fig. 5 the elements of parallel connections due to strain P1\&P6 which witness the compression and also decompression with different intensities .

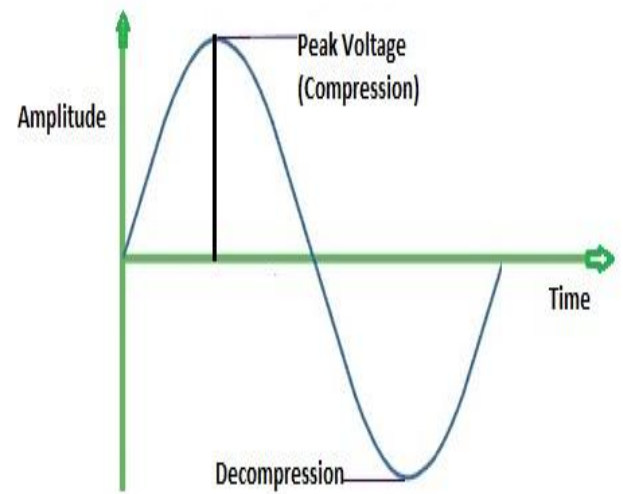

Fig.7 Similar in time domain signal of P1 \&P6

In Fig. 7 the similar in time domain signal of P1\& P6 with generation of AC signal. The same concept for the P2 \& P5 signal generator which is differ from the previous time domain signal as shown in Fig. 8.

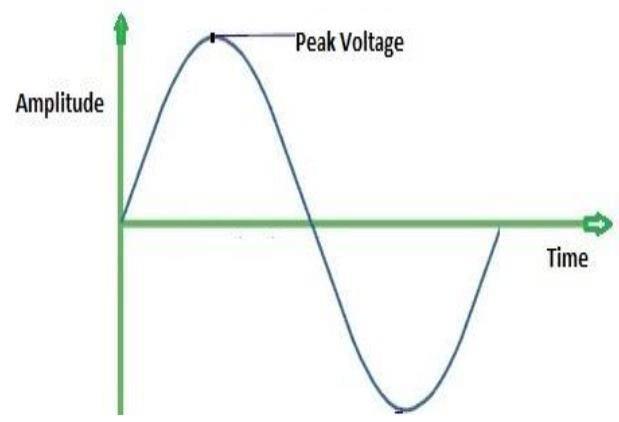

Fig.8 Similar in time domain signal of P2 \&P5

Due to the parallel connection the AC signals gets dissipated and overlapped.

\section{PROPOSED NEW DESIGN FOR ARRANGEMENT IN PIEZOELECTRIC}

The element connections should be rearranged to increase the harvester productivity. When the elements in the row face of horizontal for compression and also decompression of PZT with the same time created while vibrations walking in the piezoelectric, with this AC signal never cancel with each out other. Same elements in the same row horizontal but with a separate full wave bridge rectifier so the row output gets adding up to the DC signal and also the harvesting of energy can be boosted.

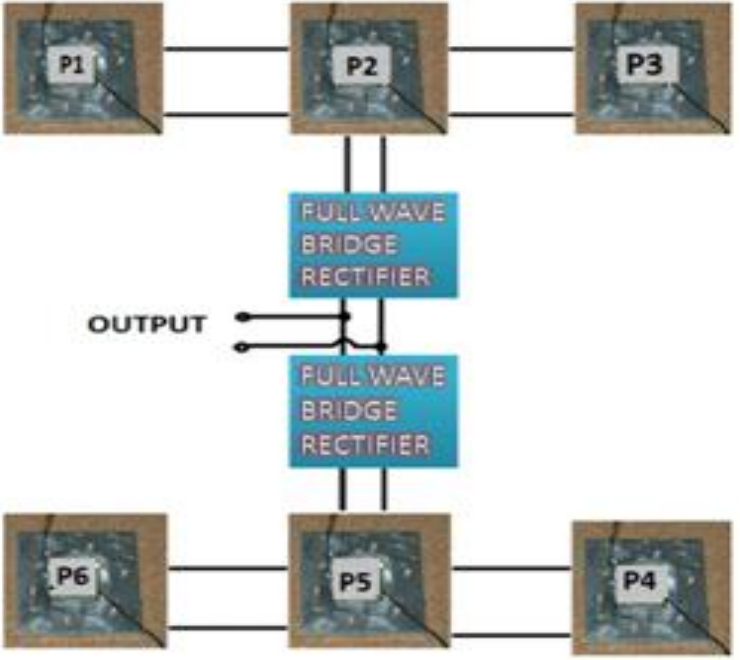

Fig.9 Proposed new Design for Arrangement in Piezoelectric

The output of power from the row of elements in horizontal gets addition as a signal of DC as shown in Fig. 9. For more output harvested powered extra elements of row arrays separately connected with full wave bridge rectifier with low voltage drop.

\section{RESULTS}

The harvester output for assumption in the piezoelectric transducer at self resonant frequency for circuit with RC resonant resonating.

$C=C_{1}+C_{2}+C_{3}$

$R=R_{1} / / R_{2} / / R_{3}$

The capacitance (equation 4) and resistance in combination (equation 5) of an piezoelectric (P1, P2,P3) array arrangement.

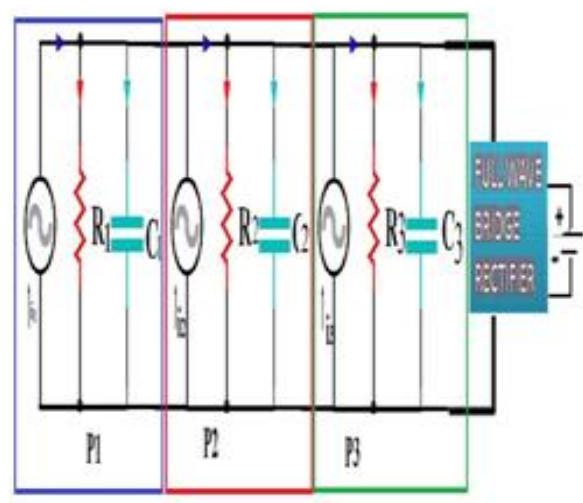

Fig.10 Elements connected in a grid

$i=i_{1}+i_{2}+i_{3}$

Equation (5) current generated by a grid as per Kirchhoff's law $P_{R}=C_{i} * V_{R} * f_{i}\left(V I-V R-2 V d_{i}\right)$

Where VI is PZT unit output open circuit voltage, Vdi is diode voltage drop, fi is transducer excitation frequency, VR is rectifier output voltage, $\mathrm{Ci}$ is piezoelectric transducer plate capacitance and PR is one piezoelectric diaphragm the total 
power output for the bridge rectifier unit The three transducers with two rows combination the total power of the grid. The total power of two rows of the circuit will be:

$P_{t}=P R_{1}+P R_{2}$

For the above mentioned circuitry the output obtained follows as:

For load connection output of rectifier VDC $\sim 1.45 \mathrm{~V}$ and Frequency $\sim 3.20 \mathrm{~Hz}$.

For no load connection output of rectifier VDC (peak to peak) $\sim 9.1 \mathrm{~V}$ and Frequency $\sim 6.41 \mathrm{~Hz}$.

Fig. 11 breadboard arrangements with circuit assembled Fig. 12 shows the waveforms which is measured with Cathode Ray Oscilloscope for without load connection. Considering load as LED is connected to the output circuit of DC rectifier.

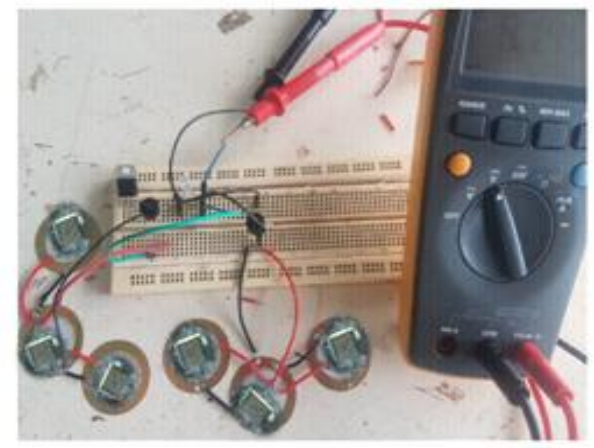

Fig.11 Energy harvesting setup for assembled test For energy harvester with the piezoelectric working test setup, can be assembled with a breadboard, same as arrangement of shown in Fig 9. VRMS is nearly equal to the 1.45V. In Fig 13 the LED output circuit is shown. LED glows with the vibrations of mechanical input to the ceramic PZT. In fig. 12 Vpeak of about $9.1 \mathrm{~V}$ output waveform at no load circuit connection.

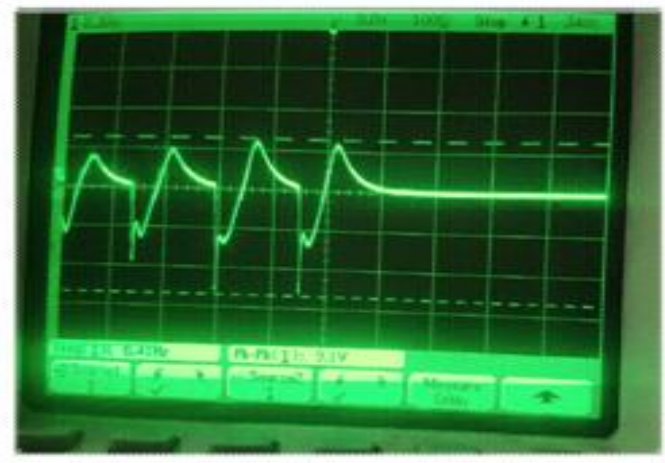

Fig.12 Oscilloscope output waveforms

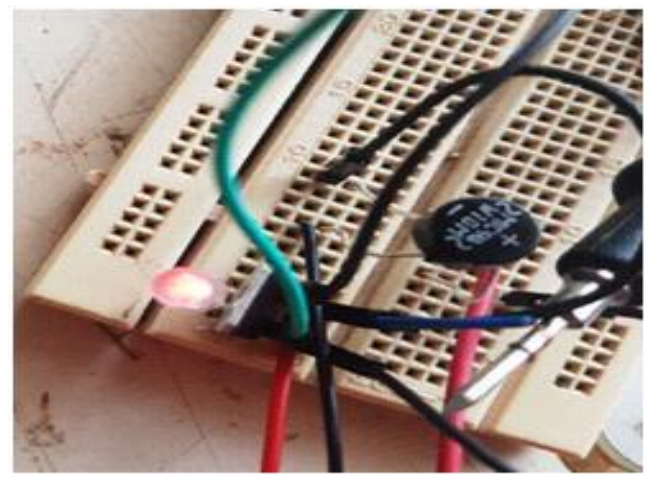

Fig.13 Load circuit connection for LED

\section{CONCLUSION}

Next upcoming years this kind of energy harvesting is going to attract the untapped market and various surrounding of energy explore other than solar energy. It will commercialize soon and also gain the popularity. For self power electronics huge demand in the upcoming years. Across the globe this concept is proved and turned out success. PZT coated bimorph transducer available now a day. So many companies developing strips of PZT rather than the square diaphragms, for energy harvesting. For the effective energy harvesting with the piezoelectric an IC LTC@3588-1 is used which gives fewer drops across rectifier with a more efficiency . Efficiency can be improved with various factors to generate the energy which is considerable. The factors like thickness of layer material, coefficient of piezoelectric, dielectric constant, PZT material used, etc. Design with a circuitry for the process of energy harvesting with a ultra capacitors fast charging and also rectifier low drop etc.

\section{REFERENCES}

1. "Conservation of energy", paper title and editor)," en.wikipedia.org/wiki/Conservation_of_energy.

2. "Thepiezoelectriceffect",www.aurelienr.com/electronique/piezo/piezo. pdf.

3. D. Kumar, P. Chaturvedi, N. Jejurikar, "Piezoelectric energy harvester design and power conditioning", 2014 IEEE Students' Conference on Electrical Electronics and Computer Science, pp. 1-6, 2014.

4. AkshayPatil, MayurJadhav, Shreyas Joshi, Elton Britto, "Energy Harvesting using Piezoelectricity", 2015 International Conference on Energy Systems and Applications (ICESA 2015), pp. 517-521, 2015."Conservationofenergy",en.wikipedia.org/wiki/Conservation_of_ energy. Adnan Mohamed Elhalwagy, Mahmoud Yousef M. Ghoneem, Mohamed Elhadidi, "Feasibility Study for Using Piezoelectric Energy Harvesting Floor in Buildings' Interior Spaces", International Conference - Alternative and Renewable Energy Quest, AREQ 2017, 1-3 February 2017,Spain, pp. 114-126, 2017.

5. J. Wang, "Fundamentals of erbium-doped fiber amplifiers arrays (Periodical style-Submitted for publication)," IEEE J. Quantum Electron., submitted for publication.

6. X. Xu et al., Application of piezoelectric transducer in energy harvesting in pavement, Int. J. Pavement Res. Technol. (2017) https://doi.org/10.1016/j.ijprt.2017.09.011

7. "Single-phase glass passivated silicon bridge rectifier", http://www.rectron.com/data_sheets/w005m-w10m.pdf

8. "PiezoelectricProducts\&EvaluationKits",www.mide.com/collections/pi ezoelectric-products

9. "ManpowerEnergyHarvestingPowerSupply",http://www.analog.com/m edia/en/technical-documentation/data-sheets/35881fc.pdf

\section{AUTHORS PROFILE}

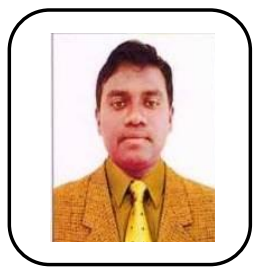

Burri Ankaiah received the B-Tech degree in electrical and electronics engineering from JNTU University, Hyderabad, and has M-Tech degree from JNTU Hyderabad, INDIA .Currently, working as Assistant Professor, School of EEE,REVA University, Bengaluru and pursuing the Ph.D. degree in electrical engineering REVA University .

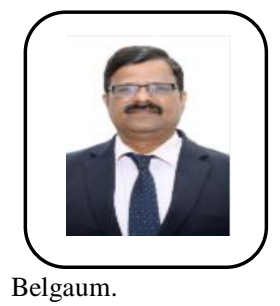

Dr. Rajashekar P. Mandi, Director, School of Electrical and Electronics Engineering, REVA University, holds Doctorate from NITK, Surathkal in the area of "Power and Energy" and holds M. Tech. degree with 3rd Rank in "Energy Systems Engineering" from BV Bhoomaraddi College of Engineering \& Technology, Hubli of Visveswaraiah Technology University (VTU),

Belgaum.

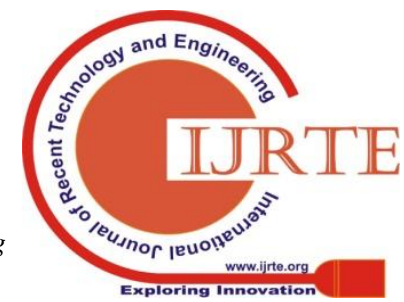


He also obtained Master of Computer Application (MCA) degree from IGNOU, New Delhi. He is a certified energy auditor from Bureau of Energy Efficiency (BEE), Govt. of India. He has three years of teaching experience. Prior to venturing into the field of academia, he had worked in Central Power Research Institute (CPRI), a premier research institute under the Ministry of Power (MoP), Govt. of India, for more than 26 years in the area of Power system simulation, Power distribution system strengthening, Power quality audit in process industries \& electrical distribution system, Electrical Safety audit in process industries, Energy conservation, Energy audit in thermal \& hydro power plants (more than 120 power plants), process industries, institutions \& buildings, Power system, Renewable energy systems, testing of Lighting equipment and solar PV systems. His research interests include smart grid, microgrid, LVDC, hybrid power system, renewable energy systems, energy conservation, strengthening of electrical distribution systems, electrical safety, power quality, LED lighting systems, etc. Presently he is guiding $8 \mathrm{PhD}$ research scholars, guided 15 MTech. Projects and 15 BTech. Projects. He had written 3 book chapters on energy conservation in Thermal Power plants and 2 book chapters in distributed power generation. He had published more than 141 technical papers in International \& Indian Journals, Conferences \& Seminars in the field of energy conservation, power quality, LED lighting system and renewable energy systems. 